\title{
CONCEPTOS, ENFOQUES Y PROPUESTAS SOBRE EL TURISMO ALTERNATIVO EN BASES DE DATOS MULTIDISCIPLINARIAS
}

\author{
Rosa María Chávez Dagostino \\ rosac@cuc.udg.mx \\ Edmundo Andrade Romo \\ edmundoa@cuc.udg.mx \\ Thania VANessa Ramos ZúnIGa \\ vanessaramos680@yahoo.com \\ Centro Universitario de la Costa \\ Universidad de Guadalajara
}

El turismo alternativo como concepto es opuesto al turismo convencional, y ha variado en el tiempo. El objetivo de este estudio fue determinar las características de la investigación en el tema a través de una revisión en Web of Science, con las palabras "turismo alternativo". Se analizó el contenido de 87 documentos con las herramientas de la plataforma. El $40 \%$ fueron publicados en los últimos tres años y conservan el ideal de sistemas turísticos más justos centrados en la comunidad. Los problemas limitantes son escasa participación de gobiernos y coordinación interinstitucional, organización comunitaria, cultura empresarial, y afluencia. El enfoque principal es el rechazo del sistema dominante del turismo.

Palabras clave: bases científicas, bibliometría, enfoque, revisión, turismo alternativo.

\section{Concepts, APPROACHES AND PROPOSALS ON ALTERNATIVE TOURISM IN MULTIDISCIPLINARY DATABASES}

The terms Alternative tourism and conventional tourism have been opposites and have evolved over time. The objective was to determine the research characteristics on this topic by searching with the term "alternative tourism" within the Web of Science. The content of 87 documents was analyzed with the tools provided by the platform. Of the 87 documents analyzed with the tools provided by this platform, 40 $\%$ were published within the last three years, and it was found that they retained the ideal of fair trade applied to tourism systems focused on the community. The limiting problems are low governmental participation and inter-institutional coordination, community organization, business culture, affluence. The main approach is the rejection from the dominant tourism system.

Keywords: alternative tourism, bibliometric, focus, review, scientific data bases. 


\section{Introducción}

—I turismo alternativo es un concepto amplio y controvertido, su auge estuvo asociado al Ereconocimiento de los impactos negativos del modelo turístico de masas, por lo que se ha considerado al turismo alternativo una forma diferente y más flexible de hacer turismo y una mejor opción para el desarrollo local. Sin embargo, su significado, los enfoques y aplicaciones, así como los objetivos de las investigaciones en torno al tema han variado a lo largo del tiempo. Su desarrollo ha estado ligado en principio al medio natural, donde luego los elementos socioculturales adquirieron relevancia, y se ha vislumbrado como un factor de desarrollo para comunidades marginadas.

Las variantes en enfoques y conceptos se dan con respecto al origen de los autores y sus instituciones, así como el tiempo en que fueron publicadas, como producto de un contexto global.

En México, el uso de este vocablo se detonó a partir de imperativos internacionales como el Año Internacional del Ecoturismo en el 2002, cuando se produjeron una serie de fascículos de turismo alternativo entre el 2000 y el 2004 (el primero de ellos con ese nombre), donde la Secretaría de Turismo (Sectur) lo promovía como el reflejo de un cambio de tendencia en el mundo, representando una nueva forma de hacer turismo, que permite al hombre un reencuentro con la naturaleza, y un reconocimiento al valor de la interacción con la cultura rural, así como una oportunidad para México de participar en el segmento con mayor crecimiento en el mercado (Sectur, 2002). Esta nueva forma de hacer turismo incluía tres segmentos, el ecoturismo, turismo rural y de aventura. Sin embargo, poco después la Sectur utilizó como sinónimo del turismo alternativo al turismo de naturaleza (Sectur, 2005), hecho que, al menos en México, ha desincentivado el uso del término en las publicaciones de los académicos. La definición del turismo de naturaleza bajo la nueva concepción de Sectur, abarca los mismos segmentos que el anterior turismo alternativo y establece que: "es una actividad económica que promueve el aprovechamiento sustentable de los recursos, una alternativa sólida para la generación de empleos, una estrategia para el desarrollo comunitario y un medio para conservar y difundir el patrimonio natural y cultural de México" (Sectur, 2017, p.6).

\section{Antecedentes}

El concepto de turismo alternativo ha sido utilizado en tantas formas que parece haber perdido sentido hablar sobre este tema, por lo que resulta más fácil referirse al segmento específico. Es un concepto en construcción, pero también en debate debido a las similitudes con otros conceptos, lo que ha conducido a ambigüedad y confusión entre los académicos.

Si bien la noción de un turismo diferente siempre ha estado presente en la historia del turismo, la aparición del vocablo parece anterior a la década de los ochenta. Por ejemplo, a finales de los setenta se discutía la incapacidad de la industria turística para representar 


\section{Conceptos, enfoques y propuestas sobre el turismo alternativo en bases de datos multidisciplinarias}

destinos como lugares reales; los sesgos y distorsiones tenían un impacto adverso en la calidad de la experiencia del visitante y en la sociedad receptora. Esta disparidad era cada vez más señalada por los críticos del turismo de masas donde la idea de lo alternativo se propone en este sentido (Britton, 1979).

En esta tesitura Holden (1984) estableció que todo lo que se aparte del turismo masivo puede etiquetarse como alternativo, y no obstante los ejemplos diversos de las alternativas que han ocurrido a la corriente principal en la historia del turismo, no lo considera un tema nuevo.

La fuerte crítica y el reconocimiento de los efectos negativos del turismo tradicional condujeron al nacimiento de un nuevo concepto de turismo, una alternativa que es una combinación de turismo de pequeña escala, "verde" e integrado a la naturaleza. Tres formas derivadas de las diferentes variaciones parecen conformar el núcleo del turismo alternativo según Triarchi y Karamanis (2017): ecoturismo, turismo cultural y turismo creativo. El ecoturismo, que jugó un papel muy importante en la década de los ochenta e implica al turismo de naturaleza, al turismo de bajo impacto y al turismo sustentable, dio paso a la forma alternativa del turismo cultural ya esbozado por Mieczkowski (1995) y por Bringas y González (2004), donde los atractivos intangibles ganaron terreno. El turismo creativo conserva las características de ambos como alternativa, pero implica que el visitante tenga una interacción educativa, emocional, social y participativa con el lugar, su cultura viva y los residentes, exige un cuidadoso diseño, respetando los principios de visibilidad, permeabilidad y flexibilidad (Triarchi y Karamanis, 2017).

Cuatro principios han sido identificados para el turismo alternativo que a su vez delinean enfoques para su estudio (Wearing y Neil, 1999) donde, a diferencia del modelo turístico de masas que promueve la gestión del cambio en términos de mercado, la solidaridad con el sistema y mantenimiento del orden social, así como la rentabilidad como indicador de la eficacia del turismo, el modelo alternativo propone un cambio radical hacia un enfoque basado en la comunidad, pretende transformar sistemas sociales, crear sistemas más justos y equitativos y, la emancipación como consecuencia.

Ante tantas variaciones y enfoques con respecto al tema, se justifica establecer los enfoques, tendencias y conceptos utilizados en la investigación relacionada con el turismo alternativo. Una de las formas de hacer evidente estos cambios en el mundo es a través de la revisión de las publicaciones científicas que se generan. Una búsqueda a través de Google Académico produce, por ejemplo, una lista de cerca de 1550000 documentos de todo tipo, en cualquier fecha e idioma, con diversos enfoques y objetivos. Esto también da cuenta de tendencias y la popularidad del tema, sin embargo, se debe ser cuidadoso en la revisión de esta información por su origen diverso y calidad.

Por otra parte, en la actualidad no existe duda de la relevancia y ventajas de la revisión y análisis de las bases de datos documentales en la innovación científica y tecnológica, en el establecimiento de tendencias, financiamiento y grupos que participan entre otros.

Existen diversas bases de datos y, ante la pregunta de qué bases de datos se deben emplear para hacer los análisis, algunas respuestas encontradas, por ejemplo, son las 
citadas por De Granda-Orive et al. (2013), donde comparativas previas entre las bases de Scopus (Elsevier, 2019) y WoS dejan claro las ventajas relativas de cada una con respecto a lo que se desea analizar, de la disciplina y del periodo de análisis, aunque también se ha establecido una gran semejanza entre ambas bases de datos, de manera que resultan complementarias.

Así, ante las preguntas de si el concepto se ha unificado, si el término es vigente, quiénes son los que investigan al respecto y sus preocupaciones, se planteó el objetivo de este trabajo de determinar los enfoques y tendencias en la investigación turística relacionada con el turismo alternativo, las variaciones en los conceptos, así como autores y sus propuestas, a través de bases de datos científicas multidisciplinarias.

Es importante señalar que la publicación en revistas internacionales de alto impacto académico contenidas en las bases reconocidas como de calidad, tiene esta ventaja, sin embargo, las limitantes para autores latinoamericanos provocan diferencias entre países e instituciones $\mathrm{y}$, una no representatividad de las investigaciones en el mundo en estas bases (Alonso-Gamboa y Reyna-Espinosa, 2016; Osorio-García, 2016), por lo que puede considerarse una limitante de este trabajo.

\section{Métodos}

Se hizo una revisión bibliográfica utilizando las bases científicas multidisciplinarias incluidas en WoS, que es un sistema integrado basado en la web, que ofrece una plataforma de contenido de alta calidad y las herramientas para acceder, analizar y gestionar información de la investigación. WoS incluye la Colección Principal de Web of Science (Science Citation Index Expanded, Social Sciences Citation Index, Arts \& Humanities Citation Index, Book Citation Index-Science, Book Citation Index-Social Sciences \& Humanities y Emerging Sources Citation Index), así como BIOSIS Citation Index, Derwent Innovations Index, $\mathrm{KCl}$-Korean Journal Database, Russian Science Citation Index, SciELO Citation Index y Current Contents Connect (Clarivate Analytics, 2018).

La búsqueda se realizó en todas las colecciones, utilizando las palabras clave de "turismo alternativo" en el título, delimitado al periodo entre 1970 y 2018. Se localizaron 182 documentos de los que se revisó el título, resumen y palabras clave con el fin de verificar el sentido en el que se utilizaron ambas palabras en los trabajos de investigación. Cuando estas no eran adyacentes, generalmente adquirían otro significado que se apartaba del interés de este trabajo por lo que se descartaron después de la revisión, el listado se redujo entonces a 87 publicaciones.

Con las herramientas de WoS se examinaron y graficaron estas publicaciones de acuerdo con el país de origen de los autores, el año y tipo.

Luego se obtuvieron los trabajos en extenso, en el caso de los artículos y libros a través de bibliotecas digitales y, en algunos casos en físico. Estos documentos se revisaron nuevamente, haciendo un análisis de contenido centrado en el resumen, objetivos, métodos y conclusiones. Se elaboró una matriz en una hoja de cálculo con los datos de autor, 


\section{Conceptos, enfoques y propuestas sobre el turismo alternativo en bases de datos multidisciplinarias}

año, objetivo, palabras clave, conceptualización y enfoque utilizado de acuerdo con las características propuestas para el turismo alternativo según Wearing y Neil (1999): "transformación de los sistemas sociales hacia sistemas más justos y equitativos, emancipación y cambio radical hacia enfoques basados en la comunidad".

\section{Resultados}

\subsection{Características de la producción académica en turismo alternativo}

El primer documento que aborda el tema de turismo alternativo en estas bases de datos fue el de Richter (1984), quien hizo una reseña de un taller de turismo alternativo en Tailandia y resalta que este fue diferente a cualquier evento sobre turismo al que había asistido, por lo sencillo y por los objetivos perseguidos, donde se investigaron las formas en que el impacto del turismo podría ser más positivo y auténtico tanto para anfitriones como invitados en el tercer mundo. Así define el alcance, propósito y la justificación de este, una forma de turismo en la que el cliente recibe alojamiento en el hogar del anfitrión y parece ofrecer una gama de oportunidades sociales y económicas, particularmente para los países en desarrollo. En este mismo año, Holden (1984) recoge los resultados del mismo taller, tal vez el primero en el mundo con ese nombre y coincide con las formas más positivas del turismo de Ritcher (1984), a través de empresas pequeñas, recorridos no comerciales que introducen a los visitantes a la cultura, más allá del mar, arena, sol y destinos sexuales.

La mayoría de los trabajos sobre el tema han sido publicados en los últimos ocho años (70 \%), donde sobresalen los años 2016-2018 (figura 1).

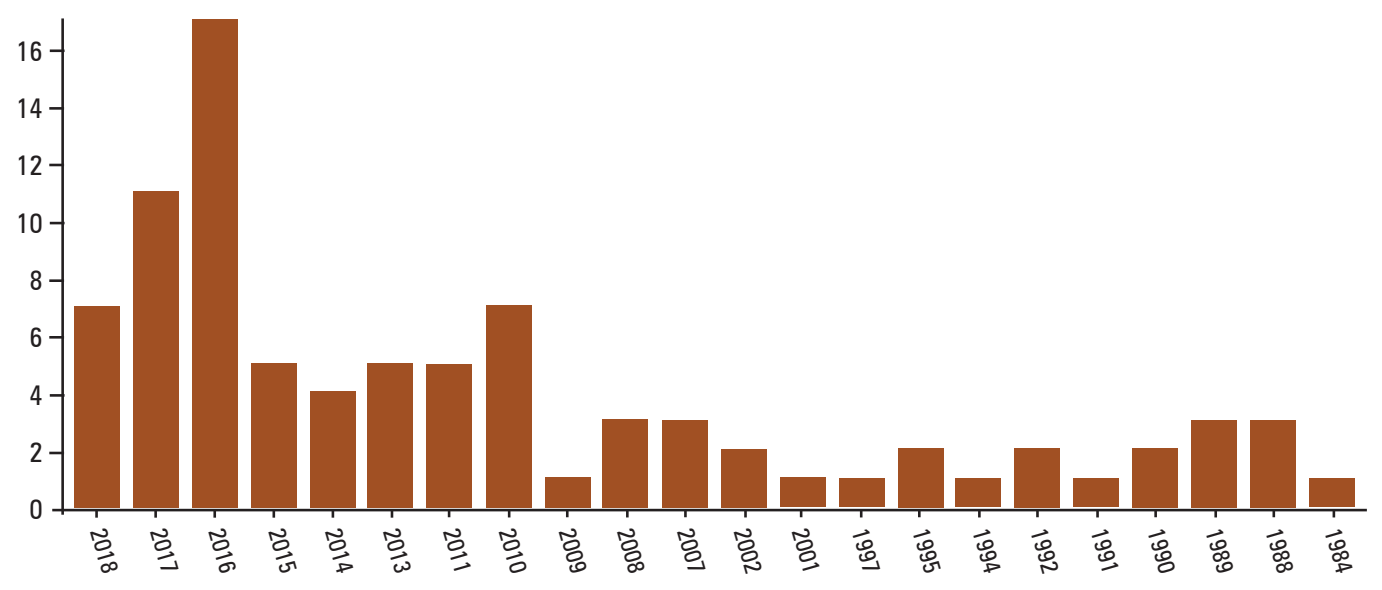

Fuente: Elaboración propia con base en las herramientas de análisis de WoS (Clarivate Analytics, 2018).

Figura 1. Número de publicaciones con el tema "turismo alternativo" en WoS por año entre 1984 y 2018 
Las áreas de investigación establecidas por WoS, representadas con mayor frecuencia con la temática de turismo alternativo, son las ciencias sociales, sociología, economía de negocios, geografía y, ciencias ambientales y ecología, donde debe aclararse, un documento puede formar parte de más de un área (figura 2).

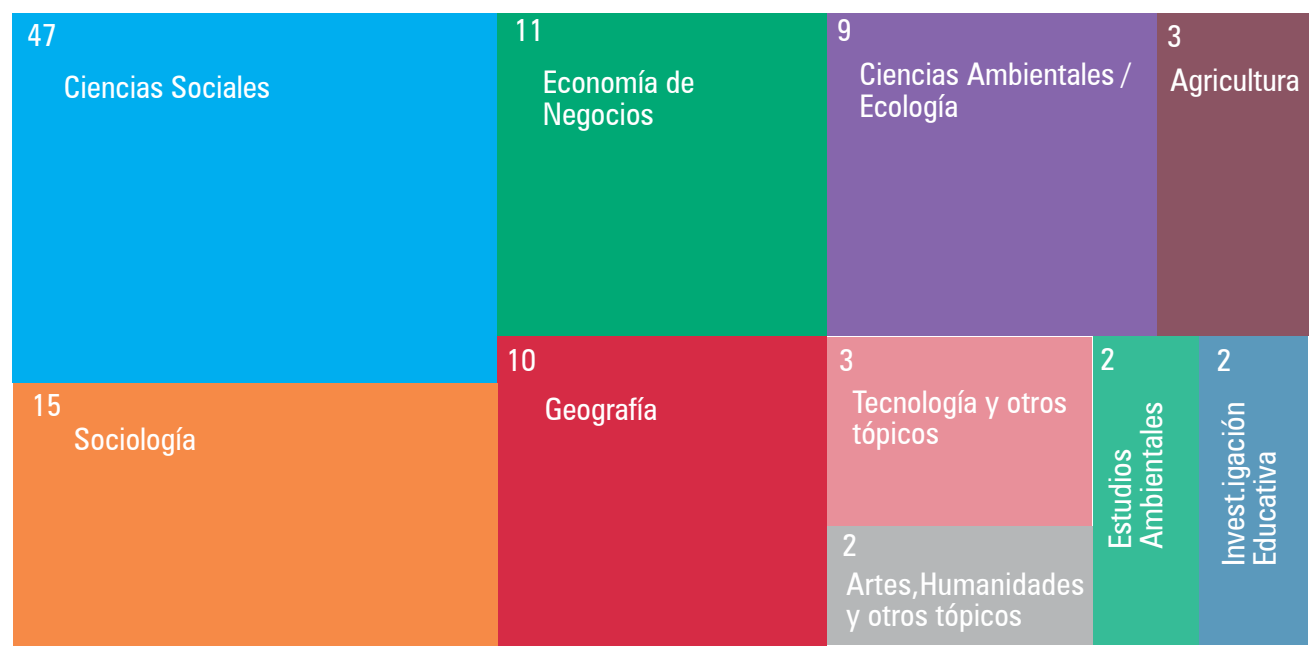

Fuente: Elaboración propia con base en las herramientas de análisis de WoS (Clarivate Analytics, 2018).

Figura 2. Áreas de investigación donde se catalogan las publicaciones sobre turismo alternativo

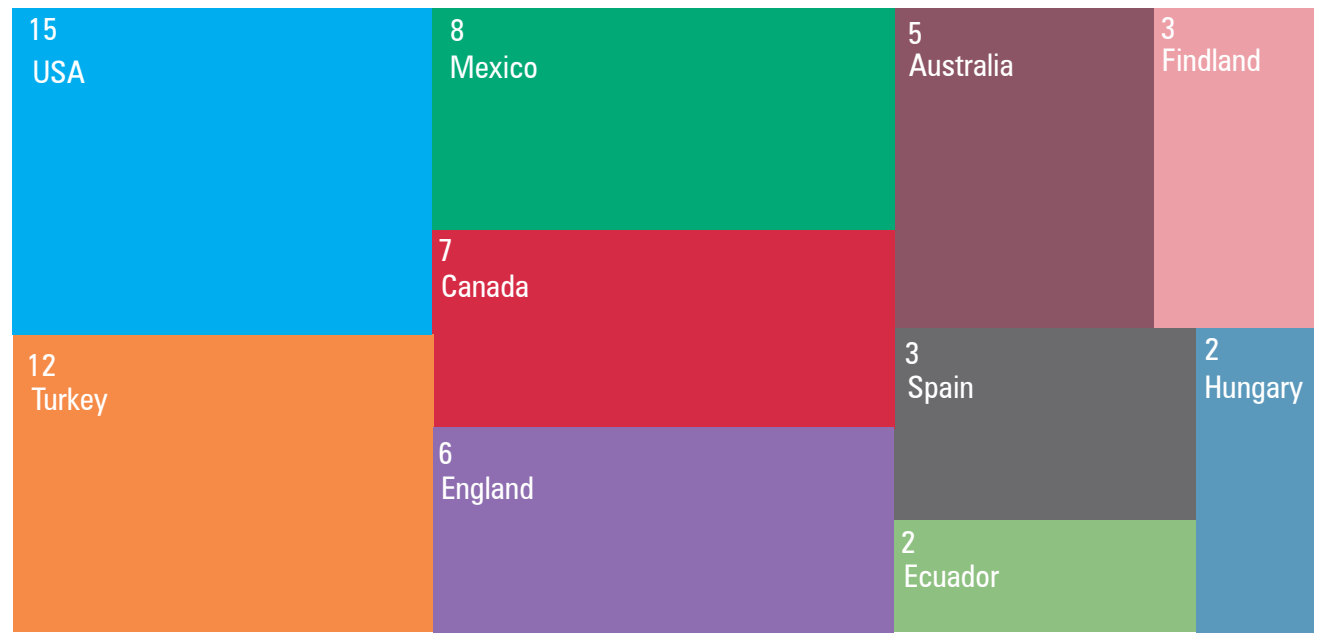

Fuente: Elaboración propia con base en las herramientas de análisis de WoS (Clarivate Analytics, 2018).

Figura 3. Principales países que participan en la producción académica relacionada con turismo alternativo 


\section{Conceptos, enfoques y propuestas sobre el turismo alternativo en bases de datos multidisciplinarias}

El tipo de documento mejor representado en las bases de datos fue el de artículo (80\%), seguido por capítulo de libro o libro (18 \%) y, el resto fue del tipo de revisión, editorial o reseña de evento.

Se encontró también que 24 países contribuyeron al tema en estas bases. Los países que dominaron el tema por la participación de al menos un coautor de ese origen, fueron Estados Unidos de América, Turquía y México. En el primer caso era de esperarse la dominancia de investigaciones de este origen según lo encontrado por otros autores en temas de turismo (Osorio-García, 2016). El caso de Turquía, que ha incursionado fuertemente en el tema, se explica por la publicación de un libro con varios capítulos centrados en el tema del turismo alternativo (Egresi, 2016b), cada uno se reflejó en las estadísticas (figura 3).

El caso de México está representado por los trabajos de Picasso-Salazar, Ruiz-Ceseña, y Torres-García, 2018, Vázquez y Lara (2018), Méndez, García, Serrano, Santos-Olmo e Ibarra (2016), Cueto-Valdivia, Flores-Vilchez, Nájera-González y Zepeda-Loera (2016), Espinoza, Bañuelos y López (2016), Pérez, Juárez , Ramírez y César (2010), Serrano-Barquín, Pérez-Ramírez, Manjarrez-Campos, y González-Melgarejo (2010) y, Osorio-García (2010).

Los artículos publicados en el 2018 versan sobre el potencial y oportunidades para desarrollar el turismo alternativo, asociado a beneficios económicos. En el 2016 se analiza el potencial de un tipo de turismo vinculado a factores culturales que podría representar una oportunidad para desarrollar turismo indígena; se determina la viabilidad del turismo alternativo en una comunidad, con base en la percepción local del recurso, la disposición para participar y la organización comunitaria, donde este último rubro resultó problemático. En el 2010 los documentos mexicanos localizados hacen énfasis en el turismo armónico como alternativa sustentable y se analiza un caso para conocer el recurso turístico aprovechado, se concluye que el turismo rural contribuye a la mejora de ingresos, y se centra en la mejora de las condiciones de vida local.

Se encontró también que estos documentos habían recibido a mediados del 2018, un promedio de 18 citas por año y 596 en total en la colección de WoS, donde el año que más citas han tenido fue en el 2017. Los artículos de mayor influencia por las veces que fueron citados en la colección núcleo de WoS fueron, en primer lugar, con 108 citas, Gursoy, Chi y Dyer (2010), que examinaron las actitudes de los residentes locales hacia el turismo masivo y el turismo alternativo, en residentes de Sunshine Coast, Australia. Los hallazgos revelan que el apoyo de la comunidad de acogida se ve afectado directamente y/o indirectamente por la percepción de diferentes factores: nivel de preocupación de la comunidad, el apego comunitario, los valores ecocéntricos, el uso de la base de recursos turísticos, el estado de la economía local y los impactos percibidos del desarrollo turístico. En segundo lugar fue el trabajo de Gard McGehee (2002), con 96 citas en esta colección, quien probó un modelo teórico basado en los componentes de las teorías de la psicología social (autoeficacia) y la movilización de recursos (redes) para explicar los cambios en la participación de los movimientos sociales entre los voluntarios de la expedición de Earthwatch (una forma clara de turismo alternativo según los autores). Se determinó que, como consecuencia de las redes establecidas durante toda la expedición, la participación en las excursiones tuvo un efecto 
significativamente positivo en las actividades de los movimientos sociales, pero no encontró que los cambios en la autoeficacia tuvieran un efecto significativo. En tercer lugar fue el trabajo de Lyons y Wearing (2008) quienes estudiaron al turismo de voluntariado como una forma diversificada del turismo alternativo, que implica turistas en viajes organizados para ayudar a un grupo social necesitado, en problemas sociales o ambientales.

El documento mexicano más influyente en el tema por el número de citas que ha recibido en WoS, fue el de Serrano-Barquín et al. (2010).

\subsection{Conceptos de turismo alternativo}

El turismo alternativo tiene sus orígenes en los años noventa, asociado con la escuela de la sostenibilidad según Cueto-Valdivia et al. (2016) y se muestra como una solución a los efectos perniciosos vinculados al turismo convencional, y en el cual se proponen algunas modalidades: el ecoturismo, el turismo rural, el de aventura, entre otros.

Picasso-Salazar et al. (2018) indican que Sectur, como principal ente regulador del turismo a nivel nacional en México, ha definido el término de turismo alternativo, como aquel que trata de dirigir su desplazamiento hacia sitios desconocidos, busca descubrir espacios apartados del turismo convencional en ambientes naturales. A diferencia del turismo masivo, el alternativo está dispuesto a pagar altos precios por la existencia de atractivos naturales y culturales únicos (Sectur, 2002).

Diferentes autores (Lantitsou, 2017; Trejo y Marcano, 2016) han concebido el turismo alternativo como aquel cuyo interés primordial es el medio ambiente y su preservación, donde juega un papel clave el equilibrio entre la biodiversidad con respecto a las prácticas humanas, al tiempo que colabora con el sustento de las poblaciones locales al diversificar las actividades económicas en áreas remotas.

Bringas y González (2004) y Pereiro (2015) (citado en Cueto-Valdivia et al., 2016), coinciden con los investigadores anteriores, estos visualizan al turismo alternativo como aquel que suscita conexiones espirituales con el tejido natural y coadyuva a atenuar la presión que se ejerce sobre el entorno y la diversidad biológica, incluso potenciar el significado de conservarlos en su estado natural como atractivos turísticos, donde el turismo alternativo es concebido como un modo de desarrollar un turismo más reflexivo, ético y educativo.

Por su parte, Oriade y Evans (2011) y Weaver (2006) (citados por Prince y loannides, 2017), integran otra variable al término "propiedad local". Estos autores expresan que el turismo alternativo se distingue por implementar prácticas a pequeña escala de control local, que brinda experiencias relacionadas con viajes educativos, estancias en granjas y ecoturismo.

Torres (1998) (citado en Vázquez Luna y Lara Rodríguez, 2018) define el turismo alternativo como una oferta turística distinta a la tradicional (sol y playa), que no posee límites espaciales, pues todas las superficies presentan un potencial turístico, como el ecoturismo, el agroturismo, el turismo deportivo, el turismo rural, cultural, de aventura, entre otros. Asimismo, el turismo alternativo persigue la optimización en el contacto entre los anfitriones y los huéspedes, lo que da como resultado interacciones de corte positivo y experiencias 


\section{Conceptos, enfoques y propuestas sobre el turismo alternativo en bases de datos multidisciplinarias}

compartidas, promoviendo la inclusión de la comunidad y la sostenibilidad en el desarrollo turístico (Eadington y Smith, 1992 y Pearce, 1992, citados en Nunkoo y Gursoy, 2017).

El turismo alternativo, o como muchos lo llaman, turismo sostenible, turismo responsable, turismo justo; es aquel que apuesta por la renovación del sistema turístico a favor de la sostenibilidad, entre cuyos intereses destacan la preservación de los entornos sociales y culturales de los diferentes destinos turísticos (Weaver y Lawton, 2002; Hiernaux, 2008; Cañizares, 2013; citados en Méndez, García, Serrano de la Cruz e Ibarra, 2016).

Para algunos autores el turismo alternativo es una tendencia re-emergente que ha sido paralela a la corriente dominante desde el principio, lo que condujo a nuevas formas (segmentos) que obedecen a numerosos factores (Calvo, Morales y Zikidis, 2017).

\subsection{Propuestas}

Las implicaciones de practicar un turismo alternativo van direccionadas a ofrecer a las áreas donde se realiza, numerosos beneficios, muchos académicos (Lantitsou, 2017; Polanco, 2017; Cueto-Valdivia et al., 2016; Espinoza et al., 2016; Vázquez Luna y Lara Rodríguez, 2018; MacDonald, 2018; Jovicic, 2016) han presentado en sus trabajos, propuestas relacionadas con los roles que debe desempeñar esta nueva modalidad de turismo, la mayoría de ellos ha coincidido en que el papel primordial del turismo alternativo es la conservación de los recursos naturales y creación de identidad y producción cultural.

Por otro lado, distintos especialistas (Egresi, 2016; Kulusjärvi, 2017; Lantitsou, 2017; MacDonald, 2018; Picasso-Salazar et al., 2018; Prince y loannides, 2017) han propuesto en sus investigaciones los beneficios del turismo alternativo, y lo han visualizado como una actividad capaz de generar un desarrollo local sostenible y autosuficiente, principalmente, en países como Ecuador (Polanco, 2017), Venezuela (Trejo y Marcano, 2016) y México. En este último, el turismo alternativo además ha sido practicado para lograr un desarrollo en comunidades indígenas que aún conservan su herencia ancestral y cultural (Cueto-Valdivia et al., 2016; Espinoza et al., 2016).

Otros autores (Özkan, Yağlı, Satılmış, Gençtürk y Yağcı, 2016; Chemli, 2017; MacDonald, 2018) expresan que a través del turismo alternativo es plausible la diversificación de productos turísticos y, de este modo, mejorar la oferta existente, algunas de las afirmaciones anteriores fueron posible gracias a estudios que se efectuaron en países como Turquía y Túnez.

No se puede dejar de lado el papel clave que juega este nuevo modelo en el fortalecimiento de procesos sociales (Sou-Yeon, 2018; Daniele, Bate y Quezada, 2017); involucramiento de diversos entes de cambio; emprendimiento social, al tiempo que promueve la política de distribución de beneficios; la construcción social de la naturaleza y el control y gestión local (Calvo, Morales, y Zikidis, 2017; Sou Yeon, 2018; Prince y loannides, 2017).

Por último, pero no menos importante, están los impactos económicos propiciados por el turismo alternativo, a pesar de que su enfoque principal es la preservación del tejido natural y el patrimonio cultural de áreas remotas, también busca impulsar todos los sectores de la economía en áreas rurales (Lantitsou, 2017), lo anterior conduce a una mejora de la calidad de vida de la población involucrada (Polanco, 2017). 


\subsection{Enfoques}

En el análisis de contenido se encontró un marcado enfoque donde el término de turismo alternativo se asocia con la transformación de sistemas sociales que se consideran injustos, donde el turismo replica estas características. Aunque los enfoques identificados no fueron explícitos en su mayoría, con base en la propuesta de Wearing y Neil (1999), en los últimos tres años (2016-2018) se encontró que los más representados fueron el de "transformar sistemas sociales" y "crear sistemas más justos" en turismo sugerido desde la idea de apartarse del turismo de masas o subrayando las bondades del turismo alternativo, creando nuevos productos turísticos como una alternativa de desarrollo comunitario o de alcanzar la sustentabilidad en destinos turísticos, entre otros (Alvear Escobar, Quishpe Fernandez, \& Enriquez Panchi, 2016; Chemli, 2017; Cueto-Valdivia et al., 2016; Egresi, 2016a; Egresi, 2016c; Espinoza López et al., 2016; Gezici \& Salihoğlu, 2016; Kulusjärvi, 2017; Nunkoo \& Gursoy, 2017; Nunkoo \& So, 2016; Opačić \& Banda, 2018; Turanlıgil, 2016).

El enfoque de "emancipación" no fue ubicado y, por último, el enfoque de "cambio radical basado en la comunidad" fue localizado en dos documentos. En este último enfoque, Ki Min (2017) propone que en la economía de mercado actual se vende y compra usando dinero, mientras que el sistema de economía compartida es un sistema económico donde las personas comparten sus bienes y servicios. A medida que surgió lo que llaman la Cuarta Revolución Industrial y la sociedad hiperconectada, la economía del intercambio está ampliando su alcance a través del intercambio de cosas y de las personas, hecho a que la industria del turismo presta atención como un modelo de turismo alternativo, que difiere del modelo de turismo de masas existente. El cambio radical también es abordado por otros autores que estudian las razones de la emergencia del turismo alternativo, lo relacionan con temas de desarrollo y pobreza, incorporando el concepto de turismo social y solidario (Calvo et al., 2017).

Con base en la información anterior, es factible indicar que existe actualmente una inclinación por asociar el turismo alternativo con factores de corte social, como los cambios generados a partir de la implementación de esta actividad en las estructuras sociales y equidad social, aunado al papel protagónico que este modelo turístico brinda a los actores de las comunidades. Sin embargo, los enfoques planteados por Wearing y Neil (1999) como tal, son pobremente discutidos o abordados por los autores, quienes presentan trabajos con objetivos centrados en nuevos productos turísticos, en las formas de turismo que preservan el ambiente y el patrimonio cultural, así como el respeto a la comunidad local, la dificultad de conciliar intereses económicos con los ambientales y la equidad social, las limitantes del turismo alternativo, como el conocimiento estratégico y los recursos humanos adecuados, entre otras. Se propuso la necesidad de conceptualizar el turismo alternativo en un foro considerando a los hospederos y visitantes para alinear esfuerzos (Prince \& loannides, 2017).

Todos estos trabajos dan cuenta de que el turismo alternativo es una forma diferente de hacer turismo sin llegar, en su mayoría, al extremo de plantear cambios radicales en el sistema turístico como base del turismo alternativo. 


\section{Conceptos, enfoques y propuestas sobre el turismo alternativo en bases de datos multidisciplinarias}

\section{Conclusiones}

El tema de turismo alternativo es abordado principalmente por países anglosajones, donde destaca Estados Unidos de América mediante artículos en idioma inglés. La participación de los países latinoamericanos en estas bases multidisciplinarias es marginal, donde el mundo académico desarrollado "del norte" domina las bases de datos de revistas científicas (Alonso-Gamboa y Reyna-Espinosa, 2016). Las causas son diversas, desde idioma y costos hasta la existencia de sistemas regionales como Latindex, Redalyc y SciELO entre otros, que han contribuido a mejorar la difusión de investigaciones latinoamericanas en español con visibilidad intrarregional (Alonso-Gamboa, 2017).

Todas las definiciones o nociones de turismo alternativo, en mayor o menor grado, comparten con distintos matices, un rechazo de lo normal, masivo o corriente principal y, una hostilidad a las políticas que privilegian capitales trasnacionales en el turismo y, en general, involucran inquietudes culturales y ecológicas de la sociedad moderna de consumo.

El vocablo y concepto (aunque todavía no consensuado) sigue siendo vigente en el mundo y con una tendencia creciente a ser estudiado, muy frecuentemente a través de estudios de caso. Sigue siendo evidente que el común denominador en las definiciones del concepto es una noción de "apartarse de los impactos negativos del turismo tradicional o de masas".

El turismo alternativo se considera un segmento que tiene antecedentes desde la década de los setenta, aunque el primer registro en bases multidisciplinarias reconocidas (WoS) fue en 1984. Este año se produjeron numerosos documentos sobre el tema, que no se reflejan en estas bases de datos.

Adquiere importancia este discurso en los años noventa asociados probablemente a otros eventos internacionales como el Informe Brundtland y la Reunión Cumbre de Río. Su origen está relacionado con la escuela de la sostenibilidad; hoy se considera una de las estrategias del turismo sustentable y se muestra como una solución a los daños ocasionados al entorno natural y cultural por el turismo convencional. Así, uno de los principales objetivos de esta modalidad es la preservación del medio ambiente y el patrimonio cultural. Este tipo de turismo también muestra un interés particular por transformar las estructuras sociales, involucrar a los pobladores locales, así como fomentar la iniciativa y gestión local para generar un desarrollo sostenible.

En los últimos tres años (2016-2018) se publicó poco más de una tercera parte de las investigaciones asociadas al turismo alternativo en las bases analizadas, donde existen diversas propuestas de académicos sobre los beneficios de llevar a cabo esta modalidad, la mayoría de los autores coincide en que este es un medio eficaz para la conservación de los recursos naturales, creación de identidad y producción cultural. Otra de las implicaciones de efectuar turismo alternativo es que es una actividad capaz de generar un desarrollo local sostenible y autosuficiente, principalmente en países de Latinoamérica, lo que fue cuestionado en la década de los ochentas. Igualmente, a través de este segmento 
de turismo es plausible la diversificación de productos turísticos para mejorar la oferta en regiones con potencial turístico.

Otras variables mencionadas en las propuestas de diferentes especialistas vinculados al papel que debe desempeñar el turismo alternativo fueron: el fortalecimiento de procesos sociales; involucramiento de diversos entes de cambio; emprendimiento social, al tiempo que promueve la política de distribución de beneficios; la construcción social de la naturaleza y el control y gestión local.

Con respecto al análisis de los enfoques más utilizados en las investigaciones de turismo alternativo se encontró que en los últimos tres años (2016-2018) fueron: transformar sistemas sociales, crear sistemas más justos y, por último, cambio radical hacia enfoques basados en la comunidad, esta pobremente representada.

\section{Referencias citadas}

Alonso-Gamboa, J. O. (2017). Sobre la publicación de revistas científicas en Iberoamérica. La Colmena, (96), 5-9. Recuperado de https://lacolmena.uaemex.mx/article/ view/9740

Alonso Gamboa, J. O., \& Reyna Espinosa, F. R. (2016). Revistas académicas mexicanas. Panorama y prospectiva. CIENCIA Ergo-Sum, 22(3), 181-191.

Alvear Escobar, A. G., Quishpe Fernandez, C., \& Enriquez Panchi, D. E. (2016). Tourism as an alternative community development in the community of Cotococha Pastaza Ecuador. Revista Publicando, 3(7), 203-217.

Bringas, N. L., \& González, J. I. I. (2004). El turismo alternativo: una opción para el desarrollo local en dos comunidades indígenas de Baja California. Economía, Sociedad y Territorio.

Britton, R. A. (1979). The image of the Third World in tourism marketing. Annals of Tourism Research, 6(3), 318-329. https://doi.org/10.1016/0160-7383(79)90106-3

Calvo, S., Morales, A., \& Zikidis, Y. (2017). Alternative tourism and the social and solidarity economy exploring social and solidarity tourism initiatives in the global south. En S. Calvo, A. Morales, \& Y. Zikidis (Eds.), Social and Solidarity Economy: The World's Economy with a Social (pp. 210-240). Routledge.

Chemli, S. (2017). Alternative tourism in a rural region, diagnosis and marketing actions. The case of the South-East of Tunisia. Korean Journal of Tourism Research, 32(4), 285-299. Recuperado de https://www.dbpia.co.kr/Journal/ArticleDetail/ NODE07226141\#

Claryvate analytics. (2018). Web of knowledge. Recuperado de https://webofknowledge. com/

Cueto-Valdivia, A. Z., Flores-Vilchez, F., Nájera-González, O., \& Zepeda-Loera, R. (2016). Turismo alternativo: una propuesta para impulsar el desarrollo en dos localidades indígenas de Nayarit, México. Teoría y Praxis, (20), 95-123.

De Granda-Orive, J. I., Alonso-Arroyo, A., García-Río, F., Solano-Reina, S., Jiménez-Ruiz, C. A., \& Aleixandre-Benavent, R. (2013). Ciertas ventajas de Scopus sobre Web of 


\section{Conceptos, enfoques y propuestas sobre el turismo alternativo en bases de datos multidisciplinarias}

Science en un análisis bibliométrico sobre tabaquismo. Revista Española de Documentación Científica, 36(2), e011. https://doi.org/10.3989/redc.2013.2.941

Egresi, I. (2016a). Alternative tourism in Turkey: Role, potential development and sustainability. Preface. En I. Egresi (Ed.), Alternative tourism in Turkey: role, potential development and sustainability (pp. VII-VIII). Cham: Springer International Publishing.

Egresi, I. (2016b). Alternative tourism in Turkey. Role, potential development and sustainability. (I. Egresi, Ed.). Estambul: Springer. https://doi.org/10.1007/978-3-319-475370

Egresi, I. (2016c). Globalization, mass tourism, and sustainable development (pp. 3-22). Springer, Cham. https://doi.org/10.1007/978-3-319-47537-0_1

Egresi, I. (2016d). Post-Fordism, alternative tourism and sustainability. En I. Egresi (Ed.), Alternative tourism in Turkey: Role, potentials, development and sustainability (pp. 271283). Cham: Springer International Publishing, A.G. https:/ / doi.org/10.1007/9783-319-47537-0_17

Elsevier. (2019). Scopus preview -Scopus- Welcome to Scopus. Recuperado de https:// www.scopus.com/

Espinoza López, P. C., Bañuelos Flores, N., \& López Reyes, M. (2016). Entre capullos de mariposas y fiestas. Hacia una alternativa de turismo indígena en El Júpare, Sonora, México. Estudios Sociales, 24(47), 312-344.

Gard McGehee, N. (2002). Alternative tourism and social movements. Annals of Tourism Research, 29(1), 124-143. https://doi.org/10.1016/S0160-7383(01)00027-5

Gezici, F., \& Salihoğlu, G. (2016). The Challenges on Sustainability of Alternative Forms of Tourism. In I. Egresi (Ed.), Alternative tourism in Turkey: Role, Potential development and sustainability (pp. 319-333). Cham: Springer International Publishing AG. https://doi.org/10.1007/978-3-319-47537-0_20

Gursoy, D., Chi, C. G., \& Dyer, P. (2010). Locals' attitudes toward mass and alternative tourism: The case of Sunshine Coast, Australia. Journal of Travel Research, 49(3), 381-394. https://doi.org/10.1177/0047287509346853

Holden, P. (1984). Alternative tourism: report of the workshop on alternative tourism with a focus on Asia, Chiang Mai, Thailand, April 26-May 8, 1984. Bangkok.

Jovicic, D. (2016). Cultural tourism in the context of relations between mass and alternative tourism. Current Issues in Tourism, 19(6), 605-612. https://doi.org/10.1080/136 83500.2014.932759

Ki Min, W. (2017). The convergent application of social science theories for the analysis of sharing economy as an alternative tourism model: Focusing on exchange theory, network theory, and actor-network theory. Journal of Tourism Studies, 29(4), 3-31.

Kulusjärvi, O. (2017). Sustainable destination development in northern peripheries: A focus on alternative tourism paths. The Journal of Rural and Community Development, 12(2/3), 41-58.

Lantitsou, K. (2017). Alternative tourism forms: the example of Drymia in Eastern Macedonia-Thrace District. Management of Environmental Quality: An International Journal, 28(3), 368-383.

Lyons, K. D., \& Wearing, S. (2008). Volunteer tourism as alternative tourism: Journeys beyond otherness. En Kevin D. Lyons \& S. Wearing (Eds.), Journeys of discovery in 
volunteer tourism : international case study perspectives (pp. 3-11). Oxxon: Cabi Publishing.

MacDonald, S. M. (2018). Sentinels by the sea: keeping as an alternative tourism performance. Text and Performance Quarterly, 38(1-2), 19-37. https://doi.org/10.1080/ 10462937.2018.1457172

Méndez Méndez, A., García Romero, A., Serrano de la Cruz Santos-Olmo, M. A., \& Ibarra García, V. (2016). Determinantes sociales de la viabilidad del turismo alternativo en Atlautla, una comunidad rural del Centro de México. Investigaciones Geográficas, 2016(90), 119-134. https://doi.org/10.14350/rig.48297

Mieczkowski, Z. (1995). Environmental issues of tourism and recreation. Lanham: University Press of America. Recuperado de https://www.cabdirect.org/cabdirect/abstract/19961802487

Nunkoo, R., \& Gursoy, D. (2017). Political trust and residents' support for alternative and mass tourism: an improved structural model. Tourism Geographies, 19(3), 318-339. https://doi.org/10.1080/14616688.2016.1196239

Nunkoo, R., \& So, K. K. F. (2016). Residents' support for tourism: Testing alternative structural models. Journal of Travel Research. https://doi.org/10.1177/0047287515592972

Opačić, V. T., \& Banda, A. (2018). Alternative forms of tourism in mountain tourism destination: A case study of Bjelašnica (Bosnia and Herzegovina). Geographica Pannonica, 22(1), 40-53. https://doi.org/10.5937/22-16621

Osorio-García, M. (2016). Calidad científica y editorial, temáticas e indicadores bibliométricos. Estudios y Perspectivas en Turismo, 25, 539-557.

Osorio García, M. (2010). Turismo masivo y alternativo. Distinciones de la sociedad moderna/posmoderna. Convergencia, 17(52), 235-259. https://doi.org/10.1016/j. diamond.2011.12.031

Özkan, G., Yağlı, K., Satılmış, Y., Gençtürk, K., \& Yağcı, C. (2016). Posıtıonıng wıth the help of geographical ınformatıon systems for alternatıve tourısm activities: Konya province example. Internatioal Journal of Ecosystems and Ecology Science, 6/4, 601-606.

Pérez Serrano, A. M., Juárez Sánchez, J. P., Ramírez Valverde, B., \& Cesar Arnaiz, F. (2010). Turismo rural y empleo rural no agrícola en la Sierra Nororiente del estado de Puebla : caso red de Turismo Rural. Investigaciones Geográficas, (71), 57-71.

Picasso-Salazar, E., Ruiz-Ceseña, F. I., \& Torres-García, A. F. (2018). Análisis de oportunidades para el desarrollo de actividades de turismo alternativo en la región norte de Baja California Sur (México). 3c Empresa: Investigación y Pensamiento Crítico, 7(1), 32-48. Recuperado de https://dialnet.unirioja.es/servlet/articulo?codigo $=6457296$

Polanco, D. (2017). $i$ Es el ecoturismo una alternativa al turismo de aventura en el cantón El Chaco? Turismo y Sociedad, 20, 151-167. https://doi.org/https://doi. org/10.18601/01207555.n20.08.

Prince, S., \& loannides, D. (2017). Contextualizing the complexities of managing alternative tourism at the community-level: A case study of a nordic eco-village. Tourism Management, 60, 348-356. https://doi.org/10.1016/j.tourman.2016.12.015

Richter, L. (1984). Alternative tourism. Annals of Tourism Research, 11(4), 626-627. https://doi.org/10.1016/0160-7383(84)90058-6 


\section{Conceptos, enfoques y propuestas sobre el turismo alternativo en bases de datos multidisciplinarias}

Secretaría de Turismo. (2002). Turismo alternativo. Una nueva forma de hacer turismo. DF: Sectur. Recuperado de: https://cedocvirtual.sectur.gob.mx/janium/Documentos/006145.pdf

Secretaría de Turismo. (2002). Turismo alternativo una nueva forma de hacer turismo. Secretaría de Turismo (vol. 1). Ciudad de México: Autor.

Secretaría de Turismo. (2005). Programa Rector de Ecoturismo, Turismo Rural, Turismo de Aventura y demás actividades de Turismo de Naturaleza 2005-2015. Mexico. Recuperado de https://cedocvirtual.sectur.gob.mx/janium/Documentos/12005.pdf

Secretaría de Turismo. (2017). Estrategia para el Impulso y Desarrollo del Turismo de Naturaleza en México. Ciudad de México: Subsecretaría de Planeación y Política Turística, Dirección General de Planeación, Dirección de Política Intersectorial. Sectur. Recuperado de: https://www.gob.mx/sectur/documentos/estrategia-para-el-impulso-y-desarrollo-del-turismo-de-naturaleza-en-mexico-138341

Serrano-Barquín, R., Pérez-Ramírez, C., Manjarrez-Campos, E., \& González-Melgarejo, L. (2010). Turismo armónico como alternativa sustentable: Para una comunidad en el estado de México. Estudios y Perspectivas en Turismo., 19(6), 970-993. Recuperado de https://apps.webofknowledge.com/full_record.do?product=UA\&search_ mode=Analyze \&qid=7\&SID=6CPEDPRRLSUodHEDb10\&excludeEventConfig $=$ ExcludelfFromFullRecPage \& page $=1 \&$ doc $=7$

Sou-Yeon, N. (2018). Representation and materiality in social construction of natural landscape: An alternative tourism case, the Jeju Olle Trail 1), 209-230.

Trejo Castro, J., \& Agusto; Marcano Navas, N. (2016). Ecoturismo y geoturismo: alternativas estratégicas para la promoción del turismo ambiental sustentable venezolano. Revista de Investigacion, 40(88), 202-228.

Triarchi, E., \& Karamanis, K. (2017). The evolution of alternative forms of tourism: a theoretical background. Business \& Entrepreneurship Journal, 6(1), 39-59.

Turanligil, F. G. (2016). Public policy and sustainable alternative tourism. En I. Egresi (Ed.), Alternative Tourism in Turkey (pp. 349-362). Cham: Springer International. https:// doi.org/10.1007/978-3-319-47537-0_22

Vázquez Luna, D., \& Lara Rodríguez, D. (2018). Percepciones contrastantes como indicadores de análisis para el turismo alternativo: Reto del desarrollo sustentable en Veracruz, México. El Periplo Sustentable, 34, 109-135.

Wearing, S., \& Neil, J. (1999). Ecotourism : impacts, potentials, and possibilities. Butterworth-Heinemann. 